\title{
Application of synchronised phasor measurements to real-time transient stability prediction
}

\author{
C.-W. Liu \\ J. Thorp
}

Indexing terms: Piecewise dynamic equivalents, Phasor measurement. Postfault transient dynamics, Synchronism

\begin{abstract}
With new systems capable of making synchronised phasor measurements there are possibilities for real-time assessment of the stability of a transient swing in power systems. In the future, online control will be necessary as operating points are pushed closer toward the margin and fast reaction time becomes critical to the survival of the system. A piecewise constant-current load equivalent (PCCLE) technique is developed which utilises synchronised phasor measurements to provide fast-transient stability swing prediction for use with high-speed control, A numerical simulation is conducted on IEEE 39-bus system equipped with ten phasor measurement units to test the effectiveness of the PCCLE technique.
\end{abstract}

\section{Introduction}

With the advent of phasor measurement units (PMUs) [1-3] capable of tracking the dynamics of an electric power system, and with modern telecommunication abilitities, utilities are becoming able to response intelligently to an event in progress. By synchronising sampling of microprocessor-based systems, phasor calculations can be placed on a common reference [3]. The magnitudes and angles of these phasors comprise the state of the power system and are used in state estimation and transient stability analysis. By communicating time-tagged phasor measurements to a central location the dynamic state of the system can be tracked in real time. An emerging application of this technology is to track the state of the system immediately following a transient event to select an appropriate remedial control action. One such real-time control strategy is already being implemented at the Florida interface [4], and others are currently under developments [5].

There are obvious differences between the real-time stability prediction problem and offline stability assessments. In conventional offline transient stability assessments, in which the critical clearing time (CCT) is to be found, there are three stages that a power system goes through: prefault, fault-on and postfault stages. In the prediction problem, the CCT is not of interest. Instead. one can monitor the progress of the transient in real time,

\section{CIEF 1995}

Paper 1975C (P9. P11), first received 20th October 1994 and in revised form 17th March 1995

C.-W. Liu is with the Department of Electric Engineering. National Taiwan University, Taipei, Taiwan, Republic of China

J. Thorp is with the School of Electric Engineering, Cornell University, Ithaca, NY 14850, USA

IEE Proc.-Gener. Transm. Distrib., Vol. 142, No. 4. July 1995 thanks to the technique of synchronised phasor measurements. Moreover, we assume in a real-time problem that protection systems for faulted transmission lines are extremely fast and the fault is removed immediately at the fault inception. By ignoring the short fault-on stage (in which the transient phasor measurements are discarded) in real time, the prediction problem only involves prefault or base case and postfault or contingency stages.

On the other hand, many transient stability assessment techniques, while simple in offline application, are too complicated for real-time use. Real-time monitoring obviates the need for some of these techniques since the system itself is solving the differential/algebraic equations. What is required is a computationally efficient way of processing the real-time measurements to determine whether an evolving event will ultimately be stable or unstable. The availability of powerful workstations in the control centre makes new approaches to the problem possible. There is a very simple answer to the problem of real-time stability prediction for simplified model. Modern workstation technology enables one to integrate the system trajectory in real time. Given a simplified model, and an initial condition (observed phasor measurements), a workstation takes just a few hundredths of a second to calculate the system trajectory one second into the future.

In an effort to reduce the computing time of integrating the differential/algebraic equation (DAE) description of postfault transient dynanmics model, the paper presents a piecewise dynamic equivalents technique, i.e. piecewise constant-current load equivalent (PCCLE), which can eliminate the algebraic equations by approximation if the load flow solution piecewise such that only internal generators buses are preserved while retaining the characteristics of the static composite loads.

\section{Postfault transient dynamics model}

An appropriately simplified postfault transient dynamics model plays a critical role in real-time stability prediction problem. A detailed model, while more accurate for prediction of a transient event over a longer window, may involve too much computation such that it can't meet real-time demand. On the other hand, too simplified a model like the classical generator with constant admittance load model is observed to give optimistic stability results [6]. In real-time stability prediction problem, the model which can give more conservative results is preferable to the model which gives optimistic results. The reason is that failure to execute special protection schemes in real time where needed can prove quitely costly [7]. It seems that a classical conservative [6] generator with the static composite constant impedance, 
constant-current and constant-power (referred to as static ZIP) load model offers a compromise between accuracy and simplicity required for real-time application. Therefore in our study we adopt the classical generator with the static ZIP load model for describing the dynamics of postfault power systems.

\subsection{Static ZIP load modelling}

We introduce the polynomial form of the static ZIP load model which is used in the development of the piecewise dynamic equivalent. A static ZIP model is a composite load which consists of constant impedance, constant current and constant power loads, in parallel. Consider a load bus, say the $j$ th bus, which is provided with voltagedependent powers $P_{l j}$ and $Q_{i j}$ represented as a polynomial function of the voltage magnitude of that bus $V_{t j}$ namely

$$
\begin{aligned}
& P_{l j}=P_{l 0}\left(a+b V_{l j}+c V_{l j}^{2}\right) \\
& Q_{l j}=Q_{l 0}\left(d+e V_{l j}+f V_{l j}^{2}\right)
\end{aligned}
$$

Here $a, b, c, d, e$ and $f$ are coefficients that express the proportion of various loads lumped together in the real and reactive power injections.

\section{Synchronised phasor measurements}

Microprocessor-based relays obtain lower level signals from the current and voltage transducers, sample the current and voltage signals, perform calculations on the samples, and reach relaying decisions. There are a number of algorithms that have been developed for protection of transmission lines, power transformers, and buses [2]. For the purpose of the measurement systems of interest here the Fourier-type algorithms are most important, that is, the Fourier-type algorithms are necessary to produce the desired measurements. Let $y(t)$ represent a voltage or current in sine-cosine form where $a(t)$ represents noiselike signals

$$
y(t)=Y_{c} \cos \omega_{0} t+Y_{s} \sin \omega_{0} t+\varepsilon(t)
$$

Estimates of the values $Y_{r}$ and $Y_{s}$ can be obtained with a Fourier calculation where there are $N$ samples per cycle or halfcycle of the fundamental frequency $\omega_{0}$ (the nominal power system frequency, 50 or $60 \mathrm{~Hz}$ ) [2]

$$
\begin{aligned}
& \hat{\Upsilon}_{c}=\frac{2}{N} \sum_{n=0}^{N-1} y(n \Delta T) \cos \omega_{0} n \Delta T \\
& \hat{Y}_{s}=\frac{2}{N} \sum_{n=0}^{N-1} y(n \Delta T) \sin \omega_{0} n \Delta T
\end{aligned}
$$

If $y(t)$ is a pure sinusoid that equals $\cos \left(\omega_{0} t+\delta\right)$, the complex number $\hat{Y}$ computed from eqns. $a$ and $b$ has the angle $\delta$. If $y(t)$ is a bus voltage the resulting complex voltage phasor can be thought as the state of the system for many applications. As the window of $N$ samples moves in time [the sums in eqns. $a$ and $b$ taken from $n=k$ to $n=N+k-1]$ the angle of $\hat{Y}$ rotates. A reference angle can be established and the calculations made recursively by writing the equation with $\phi$ equal to $\omega_{0} \Delta T$ and $\hat{Y}^{L}$ as the phasor computed using $N$ sample values ending at sample $L$

$$
\hat{Y}^{L}=\hat{Y}^{L-1}+\left(\frac{2}{N}\right)\left[y_{L}-y_{L-N} \exp (j N \phi)\right] \exp (-j L \phi)
$$

The recursive calculation is computationally efficient since only one multiplication is performed in eqn. $c$. Even more importantly, if the signal $y(t)$ is a pure sinusoid at the nominal frequency $\omega_{0}$, then $\hat{\gamma}^{L}$ is stationary in phase with a phase angle equal to the angle $\delta$ at the instant at which the recursion was begun. If the frequency is $\omega_{0}$ plus $\Delta \omega$ (the power system is off nominal frequency because of a generation-load imbalance), the computed phasor rotates at a rate $\Delta \omega$ providing a highly accurate freqency measurement device, that is, the frequency can be measured by watching the computed phasor rotate. It is assumed that the sampling rate is fixed, i.e. $\Delta T$ equals $2 \pi / \omega_{0}$.

Currently, one limiting factor to this technology is the availability of an accurate sampling clock synchronism system. The use of a navigation broadcast system such as the global positioning system (GPS) has made it possible to produce synchronising pulses once every second with accuracy of $1 \mu \mathrm{s}$. With such accuracy it is possible to obtain accurate voltage phasor measurements during fast changes of system operation such as transient swings.

\section{Criteria for transient stability prediction}

This Section sets up the criteria for stability identification of a developing swing. Traditionally, transient stability is concerned with the capability of generators to capture synchronism following a large disturbance, such as threephase short circuit, loss of generator or sudden change of load, etc. In this case, the criterion can be so defined that a swing after a fault is determined as an unstable swing if the relative angles of different generator groups separate indefinitely with time. Otherwise, it is identified as a stable swing. However, in practice, a postfault swing which doesn't cause synchronism problem may exhibit pole slipping, voltage dip and overloaded transmission line phenomena. These conditions may initiate important relay operations such as out-of-step blocking and tripping causing undesirable situation. Therefore a more stringent stability criterion is needed to meet real-time operation requirements, and we define the practical transient stability criterion in terms of security constraint region in state space for prediction use.

\subsection{Generator angle security constraint}

Considerations of out-of-synchronism and pole-slipping conditions impose limits on the machine angles of internal buses with respect to the slack bus (the $N_{\theta}$ th bus) $\delta_{k}$ as

$$
\left|\delta_{k}\right| \leqslant \pi
$$

Denote the security region inside the limits by $R_{b}$, i.e.

$$
R_{\delta}:=\left\{\delta_{k}:\left|\delta_{k}\right| \leqslant \pi\right\}
$$

\subsection{Generator frequency deviation constraint}

The frequency deviation that accompanies system disturbances is caused by the imbalance between load and generation. Excess generation will cause a rising frequency; excess load, however, will cause the frequency to drop. Both cases will cause permanant damage to steam turbines. Therefore the limits are imposed on the generator frequency deviation $\omega_{k}$ as

$$
\left|\omega_{k}\right| \leqslant \alpha
$$

Denote the region inside the limits by $R_{\omega}$, i.e.

$$
R_{\omega}:=\left\{\omega_{k}: \Delta \omega_{k} \leqslant \alpha\right\}
$$

IEE Proc.-Gener. Transm. Distrib., Vol. 142, No. 4, July 1995 
4.3 Load bus voltage magnitude security constraint Considerations of operating and voltage dip impose the limits on load bus voltage magnitude $V_{k}$ as

$$
\left|V_{k}-1\right| \leqslant \varepsilon
$$

Denote the corresponding security region in space $V$ by $R_{V}$, i.e.

$$
R_{V}:=\left\{V_{k}:\left|V_{k}-1\right| \leqslant \varepsilon\right\}
$$

\subsection{Load bus voltage angle security constraint}

Thermal considerations limit the amount of current flowing through transmission lines and transformers. By some calculations and approximations [8], the line flow constraints may be expressed approximately in terms of phase angle differences. It implies there are limits imposed on load bus voltage angles $\theta_{k}$ as

$$
\left|\theta_{k}\right| \leqslant \beta
$$

Denote the security region in $\theta$ space by $R_{\theta}$, i.e.

$$
R_{\theta}:=\left\{\theta_{k}:\left|\theta_{k}\right| \leqslant \beta\right\}
$$

\subsection{Practical transient stability security constraint} region

We define the set in $(\delta, \omega, \theta, V)$-space

$$
R_{\mathrm{TS}}:=R_{\delta} \times R_{\omega} \times R_{\theta} \times R_{v}
$$

the practical transient stability security constraint region. With the preceding concepts, we formulate the real-time transient stability prediction problem in the following precise way.

Problem: Given a set of real-time measurement vectors $\delta(k \Delta T), \omega(k \Delta T), \theta(k \Delta T), V(k \Delta T)$ where $k=1,2, \ldots$, $M, \Delta T$ is the sampling period, and $M \Delta T$ is the length of the observation window after a fault. For $k=0$ the measurement vector comprises the prefault steady state. For $k=1,2, \ldots, M$ the measurement vectors comprise the postfault observed dynamic states. Can one find some methodology that can process the observed real-time measurements to calculate the time history of $\delta(k \Delta T)$, $\omega(k \Delta T), \quad \theta(k \Delta T), \quad V(k \Delta T), \quad k=M+1, \quad M+2$,

$M+N$, over a predicton window with length $N \Delta T$ before the transient swing actually occurs such that one can predict whether a developing swing is stable or not by the following criteria?

Stable swing: $(\delta(k \Delta T), \omega(k \Delta T), \theta(k \Delta T), V(k \Delta T)) \in R_{T s}$ for $k=M+1, M+2, \ldots, M+N$, otherwise the swing is determined as unstable one.

Remark: In practice, there are uncertainties in the model and noise in the real-time measurements, and it is safer to predict the stability of a swing by processing successive observed measurements as different initial conditions and going through the methodology in turn. Check the outcome of stability prediction. If the number of stability status is greater than the instability status, the swing is identified as stable swing. Otherwise, it is determined as unstable swing

\section{Piecewise constant current load equivalent} (PCCLE) technique

We derive the piecewise equivalencing technique which can eliminate the algebraic equation such that only internal generator buses are preserved while retaining the characteristics of composite loads approximately. More specifically, this is achieved by representing the composite loads with constant-current loads piecewise, which are linear loads in each piece, and then, by circuit theory, the load-flow solution is of closed form and the effects of load current injections can be reflected on internal generator current injections for swing equation use. Whenever the generator angles move beyond the boundary of the moving hypercube (which defines the piece in generator angle space) centred at previous base point, we update the load current injections by solving the load flow equations. The piecewise linear approximation of the static ZIP load model has also been suggested in Reference 9. The difference between that and the PCCLE is that Reference 9 approximates the equivalent current injection by piecewise constant values while here the ZIP loads are approximated by piecewise constant-current sources.

The following example illustrates the concepts. The sample power system consisting of two generators and several composite loads can be reduced to two generators, each of which has an additional current injection at its bus, accounting for the effects of load current injection, as shown in Fig. 1. The two additional current injections are functions of the machine angles $\delta_{1}$ and $\delta_{2}$. The

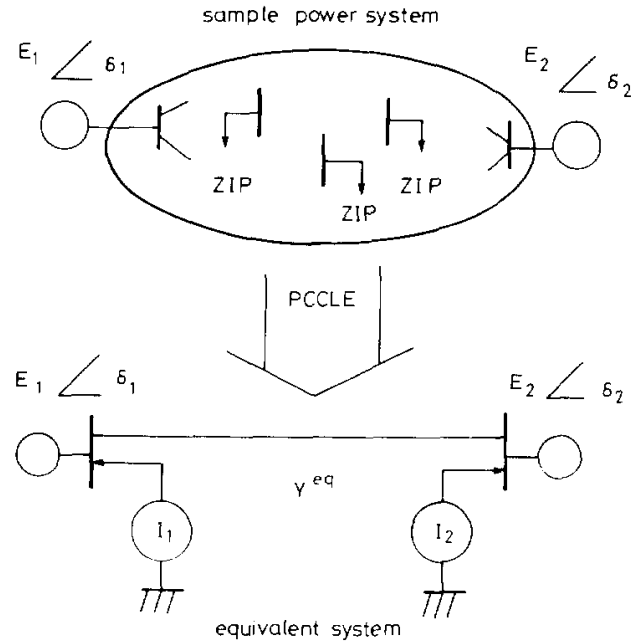

Fig. 1 Sample power system and PCCLE equivalencing technique

following are details of piecewise equivalencing process. For the original system, the bus current phasors are related to the bus voltage phasors and the bus admittance matrix $Y$ through

$$
\left[\begin{array}{l}
I_{g} \\
I_{t}
\end{array}\right]=\left[\begin{array}{ll}
Y_{g g} & Y_{g I} \\
Y_{l g} & Y_{l l}
\end{array}\right]\left[\begin{array}{l}
V_{g} \\
V_{l}
\end{array}\right]
$$

where the subscript $/$ denotes the load buses to be eliminated and the subscript $g$ denotes the internal generator buses that are preserved. After simple algebraic manipulations, the current-voltage relationship (eqn. 3) reduces to

$$
I_{g}=Y^{e q} V_{g}+D^{e q} I_{l}
$$

where

$$
\begin{aligned}
& Y^{e q}=Y_{g g}-Y_{g l} Y_{l l}^{-1} Y_{l g} \\
& D^{e q}=Y_{g l} Y_{l l}^{-1}
\end{aligned}
$$


Eqn. 4 has two terms; the first represents the contributions to generator current injections from generator voltages, and the second represents the load current's contributions. By using eqn. 4 , the swing equations for the $i$ th generator can be readily derived

$$
\begin{aligned}
& \hat{\delta}_{i}=\omega_{i} \\
& \dot{\omega}_{i}=\frac{1}{m_{i}}\left(P_{m i}-d_{i} \omega_{i}-P_{i}\right)
\end{aligned}
$$

where

$$
\begin{aligned}
P_{i}= & \operatorname{Re}\left(V_{g l} I_{g i l}^{*}\right) \\
= & V_{g i} \sum_{j=1}^{N_{g}-1} Y_{i j}^{e q} V_{g i} \cos \left(\delta_{i}-\delta_{j}-\phi_{i j}^{e q}\right) \\
& +V_{g i} \sum_{j=N_{g}+1}^{N} D_{i j}^{e q} I_{i j}(\delta) \cos \left(\delta_{i}-\varphi_{l j}(\delta)-\psi_{i j}^{e q}\right)
\end{aligned}
$$

and $Y_{i j}^{e q} \angle \phi_{i j}^{e q}$ are the entries of matrix $Y^{e q}$ and $D_{i j}^{e q} \angle \psi_{i j}^{e q}$ are the entries of matrix $D^{e q}$. Note that the load current $I_{l} \angle \varphi_{l}$ is in fact a function of generator angles due to the composite loads. The principle of the technique is that we approximate the load current that is a function of generator angles piecewisely by constant value whenever the generator angles lie inside the moving hypercube along the projected trajectory on generator angle space. The hypercube is a set of generator angles defined in angle space as follows

$$
\left\{\delta_{i}:\left|\delta_{i}-\delta_{i}^{0}\right| \leqslant \gamma\right\}
$$

where the $\gamma$ is an update parameter with unit radian or degree chosen by monitors and $\delta_{i}^{\circ}$ is the angle of base trajectory point. Fig. 2 illustrates the moving hypercubes

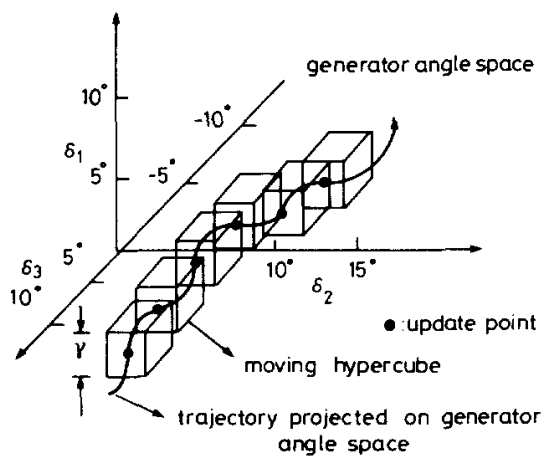

Fig. 2 Moving hypercubes in PCCLE technique

along the trajectory. With the approximation of constant current load in the moving hypercube and update of currents whenever exceeding the hypercube, the transient dynamics is then described by piecewise swing eqns. 7 and 8 . When the generator angles move beyond the boundary of the current hypercube, the constant load current will be recomputed by the following procedures.

First, choose the current generator angles as the new centre of moving hypercube and solve load flow for load bus voltage magnitudes and angles $V_{l j}, \theta_{l j}$ at a given load bus, say the $j$ th bus. Then, the new constant load current at the $j$ th bus is computed as

$$
I_{l j} \angle \varphi_{l j}=\frac{\sqrt{ }\left(P_{j}^{2}+Q_{j}^{2}\right)}{V_{l j}} \angle\left(\theta_{l j}-\phi_{l j}\right)
$$

where $\phi_{l j}$ is the power factor angle at bus $j$ given by $\varphi_{i j}=\tan ^{-1}\left(Q_{j} / P_{j}\right)$. This completes the equivalencing process.

In the remainder of this Section, we describe the piecewise constant-current load equivalent (PCCLE) technique for real-time transient stability prediction. Basically, there are two kinds of computations used in PCCLE, namely, fourth-order Runge-Kutta integration and decoupled power flow calculation. The Runge-Kutta integration is used to integrate the piecewise swing eqns. 7 and 8. The decoupled power flow method is used for updating equivalent load current injections whenever the generator angles exceed the boundary of the moving hypercube. Since the load bus voltages (magnitudes and angles) do not appear explicitly in the swing equations, they must be updated using a sensitivity analysis.

First the quantities $Y^{e q}, D^{e q}$ and the load currents at the prefault stage $I_{1}^{0}$ are computed and stored in memory to save CPU time in the PCCLE technique. However, from eqns. 5 and $6, Y^{e q}$ and $D^{e q}$ are products of postfault network parameters, that is, they are not known at the prefault stage. One way to overcome this problem is to compute various $Y^{\text {eq }}$ and $D^{\text {eq }}$ for every possible postfault network configuration and store them in memory so that the specific one can be retrieved for prediction calculation whenever it is needed. Since the postfault configuration is either the same as the prefault system or is different from the prefault system only by a tripped line, the size of memory for storing these quantities does not need to be large in a typical power system.

The load bus voltage can be computed by solving the linearised load flow equations as follows. By linearising the load flow equations about the base trajectory point centred in the current hypercube, the real and reactive power increments can be expressed in terms of the voltage magnitude increments, the phase angle increments and the Jacobian matrix $J$. In matrix form

$$
\left[\begin{array}{l}
\Delta P_{g} \\
\Delta P_{l} \\
\Delta Q_{g} \\
\Delta Q_{l}
\end{array}\right]=\left[\begin{array}{llll}
J_{11} & J_{12} & J_{13} & J_{14} \\
J_{21} & J_{22} & J_{23} & J_{24} \\
J_{31} & J_{32} & J_{33} & J_{34} \\
J_{41} & J_{42} & J_{43} & J_{44}
\end{array}\right]\left[\begin{array}{c}
\Delta \delta_{g} \\
\Delta \theta_{l} \\
\Delta V_{g} \\
\Delta V_{l}
\end{array}\right]
$$

By assuming that constant generator voltage magnitudes $\Delta V_{g}=0$, the eqn. 9 reduces to

$$
\left[\begin{array}{l}
\Delta P_{l} \\
\Delta Q_{l}
\end{array}\right]=\left[\begin{array}{ll}
J_{22} & J_{24} \\
J_{42} & J_{44}
\end{array}\right]\left[\begin{array}{l}
\Delta \theta_{l} \\
\Delta V_{l}
\end{array}\right]+\left[\begin{array}{l}
J_{21} \\
J_{41}
\end{array}\right] \Delta \delta_{s}
$$

Then perform a first-order Taylor series expansion of the static composite load model described by eqns. 1 and 2 to get

$$
\Delta P_{l j}=P_{l j}^{\prime} \Delta V_{l j} \quad \Delta Q_{l j}=Q_{l j}^{\prime} \Delta V_{l j}
$$

where

$$
P_{l j}^{\prime}=\frac{\partial P_{l j}}{\partial V_{l j}} \quad Q_{l j}^{\prime}=\frac{\partial Q_{l j}}{\partial V_{l j}}
$$

In matrix form

$$
\left[\frac{\Delta P_{l}}{\Delta Q_{l}}\right]=\left[\begin{array}{c}
P_{l}^{\prime} \\
Q_{i}^{\prime}
\end{array}\right] \Delta V_{l}
$$

Substituting eqn. 11 into eqn. 10 obtains

$$
\left[\begin{array}{l}
\Delta \theta_{l} \\
\Delta V_{l}
\end{array}\right]=G \Delta \delta_{g}
$$


where

$$
G=-\left[\begin{array}{ll}
J_{22} & J_{24}-P_{l}^{\prime} \\
J_{42} & J_{44}-Q_{l}^{\prime}
\end{array}\right]^{-1}\left[\begin{array}{l}
J_{21} \\
J_{41}
\end{array}\right]
$$

Since a load flow is performed at each update of load current in the PCCLE method, the Jacobian matrices in eqn. 9 are available for the base trajectory point in the current hypercube. It is typical, however, to compute $G$ at the prefault operating point. This does not result in a severe loss of accuracy, whereas the savings in CPU time are considerable. Thus eqn. 12 expresses the load bus voltage magnitude and angle increments as a known function of the generator angle increments. Therefore the load bus voltage magnitude and angle variations can be monitored in terms of the generator angles within the hypercube.

The PCCLE technique for real-time transient stability prediction can be summarised as follows.

Step 1: Set up the practical transient security region, $\boldsymbol{R}_{\text {TS }}$ and update parameter $\gamma$. Then compute quantities $Y^{e q}, D^{e q}$ and $I_{i}^{0}$ at prefault steady operating condition.

Step 2: Integrate the swing equations (eqns. 7 and 8) and solve the linearised load flow equation (eqn. 12) one step ahead by using arrived phasor measurement. Check whether the one-step prediction value $(\delta(\Delta T), \omega(\Delta T)$ $\theta(\Delta T), V(\Delta T))$ lies in $R_{T S}$ and predict instability in case it does not. If instability has not occurred, the one-step prediction value is checked to see whether it exceeds the boundary of the current hypercube. If it does, recompute the load currents and update the swing equations. Otherwise, no update is made.

Step 3: Repeat step 2 until the prediction interval is finished or else instability is determined.

\section{Simulation results}

This Section presents numerical simulations of the proposed PCCLE technique on the IEEE 39-bus power system. The programs are coded in FORTRAN and implemented on an HP $9000 / 720$ workstation. There are two important metrics, CPU time and approximation error, for evaluating the effectiveness of the proposed technique for the real-time prediction problem. The CPU time was measured by placing timing routine calls within the program. The first call was placed before the postfault computation (note including the computation of the prefault quantities like $Y^{e q}, D^{e q}$ and $I_{l}^{0}$ ) and the second cal was placed at the end. The approximation errors were measured by following formula

$$
\left.E=\max _{k \in \text { prediction interval }} \| \delta(k \Delta T)-\bar{\delta}(k \Delta T)\right) \|
$$

where $\delta(k \Delta T)$ is the generator angle calculated by the PCCLE technique, and $\bar{\delta}(k \Delta T)$ is the generator angle calculated by ETM simulation package [10]. We regard the generator angles calculated by the ETMSP as the 'accurate value' compared to generator angles calculated by the PCCLE technique. The justification is obvious because the ETMSP did not make any approximations in the adopted composite load model.

The IEEE ten-machine 39-bus system is used to illustrate the performance of our piecewise technique. The one-line diagram of this system equipped with ten phasor measurement units is shown in Fig. 3. The system is provided with constant $P Q$ loads. Although this system is not very large in size, it is a good example of a reduced. order system in an interconnected network. This system represents the $345 \mathrm{kV}$ transmission network of New
England [11]. Generator 10 is an equivalent power source representing parts of the US-Canadian interconnected system.

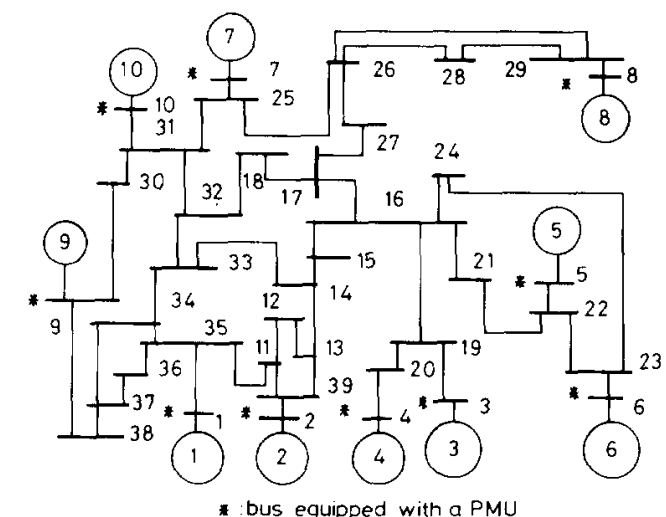

Fig. 3 One-line diagram of ten-machine system

* Bus equipped with PMU

Three-phase short-circuit-to-ground faults are simulated to occur on various transmission lines. The fault duration is two cycles followed by tripping the appropriate circuit breakers. The postfault system configuration is the same as the prefault system except that the faulted line is eliminated. We timed the execution time of predicting postfault transient swings one second into future and measured the approximation errors. The technique was tested with different update parameters on an HP 9000 / 720 workstation. The simulation results are shown in Table 1 and 2 from which we observe that PCCLE can

\begin{tabular}{|c|c|c|c|}
\hline \multirow{2}{*}{$\begin{array}{l}\text { Fault } \\
\text { location } \\
\text { Bus }\end{array}$} & \multirow{2}{*}{$\begin{array}{l}\text { ETMSP } \\
\text { CPU } \\
\text { time }\end{array}$} & \multicolumn{2}{|c|}{$\begin{array}{l}\text { PCCLE } \\
\text { CPU time }\end{array}$} \\
\hline & & $\gamma=5^{\circ}$ & $\gamma=10^{\circ}$ \\
\hline & (s) & (s) & (s) \\
\hline $11-12$ & 0.89 & 0.16 & 0.14 \\
\hline $13-14$ & 0.90 & 0.17 & 0.16 \\
\hline $15-16$ & 0.87 & 0.17 & 0.16 \\
\hline $33-34$ & 1.06 & 0.2 & 0.16 \\
\hline $36-37$ & 1.16 & 0.2 & 0.16 \\
\hline
\end{tabular}

Table 1 : Comparison of CPU time of PCCLE with ETMSP

Table 2: Approximation errors of PCCLE technique

\begin{tabular}{lll}
\hline \multirow{2}{*}{$\begin{array}{l}\text { Fault location } \\
\text { bus }\end{array}$} & \multicolumn{2}{c}{ Error (deg.)(\%) } \\
\cline { 2 - 3 } & $y=5^{\circ}$ & $y=10^{\circ}$ \\
\hline $11-12$ & $8.57(6.5)$ & $15.52(12)$ \\
$13-14$ & $8.19(6.3)$ & $17.8(13.6)$ \\
$15-16$ & $4.9(3.8)$ & $12.83(9.8)$ \\
$33-34$ & $6.62(4.7)$ & $15.87(11.3)$ \\
$36-37$ & $2.87(2.0)$ & $5.99(3.9)$ \\
\hline
\end{tabular}

predict the swings in real time with an acceptable degree of accuracy. For example, the approximation errors of $\operatorname{PCCLE}\left(\gamma=5^{\circ}\right)$ with step size $(0.02 \mathrm{~s})$ are less than $10^{\circ}$.

\section{Conclusion}

Current financial and environmental trends have forced a power system to operate under more stressed conditions than in the past. The transient stability prediction method discussed in this paper is designed to provide 
timely information for power system control in real time. The proposed PCCLE technique shows the promising simulation results for real-time transient stability prediction problem. Further investigation such as the utilisation of the PCCLE technique to effectively operate control devices need to be conducted.

\section{References}

1 THORP, J.S., PHADKE, A.G., HOROWITZ, S.H., and BEGOVIC M.M. : 'Some applications of phasor measurements to adaptive protection', IEEE Trans., 1988, PWRS-3, (2), pp. 791-798

2 PHADKE, A.G., and THORP, J.S.: 'Computer relaying for power 2 PHADKE, A.G., and THORP, J.S.: 'Com
systems' (Research Studies Press, 1988)

3 PHstems'(Research Studies Press, 1988 ) measurement technique for tracking voltage phasors, local system frequency and rate of change of frequency', IEEE Trans., 1983, PAS-102, (5), pp. 1025-1038

4 CENTENO, V., DE LA REE, J., PHADKE, A.G., MICHEL, G. MURPHY, J., and BUMETT, R.: 'Adaptive out-of-step relaying using phasor measurement techniques', IEEE Comput. Appl. Power, 1993, 6, (4), pp. 12-17

5 DENYS, PH., COUNAN, C., HOSSENLOPP, L., and HOLWECK, C.: 'Measurement of voltage phase for the French future defence plan against losses of synchronism', IEEE Trans. Power Delivery, 1992, (1), pp. 62-69

6 KENT, M.H., SCHMUS, W.R., MCCRFACKIN, F.A., and WHEELER, L.M.: 'Dynamic modeling of loads in stability studies', IEEE Trans., 1969, PAS-88, (5), pp. 756-763

7 '1988 System Disturbances'. North American Electric Reliability Council, 1989

8 WU, F.F., and KUMAGAI, S.: 'Steady state security regions of power systems', IEEE Trans., 1982, CAS-29, pp. 703-710

9 BALDWIN, T., MILI, L., and PHADKE, A.G.: 'Dynamic ward equivalents for transient stability analysis', IEEE Trons., 1994, PWRS-9, (1), pp. 59-67

10 'Extended transient/midterm stability program'. ETMSP simulation package developed from EPRI project RP 1208-9, 1990

11 HAQUE, M.H., and RAHIM, A.H.M.A.: 'Determination of first swing stability limit of multimachine power systems through Taylor series expansion', IEE Proc. C, 1989, 136, (6), pp. 373-379 